\title{
Bioimpedance in monitoring of effects of selective serotonin reuptake inhibitor treatment
}

This article was published in the following Dove Press journal:

Psychology Research and Behavior Management

29 June 2011

Number of times this article has been viewed

\section{Vasiliy Grigorievich Alexeev \\ Ludmila Vasilievna \\ Kuznecova}

Department of Physiology, SP Botkin Moscow City Clinical Hospital,

Moscow, Russia
Correspondence: VG Alexeev SP Botkin Clinic and Hospital, 2nd Botkinsky Proezd, 125284 Moscow, Russia Tel +74959450045 Fax+74959450045

Email info@botkinmoscow.ru
Background: Bioimpedance has been shown to be a safe technique when used in a number of biomedical applications. In this study, we used the Electro Interstitial Scan (EIS) to perform bioimpedance measurements to follow up the efficacy of selective serotonin reuptake inhibitor (SSRI) treatment in subjects diagnosed to have major depressive disorder.

Methods: We recruited 59 subjects (38 women, 21 men) aged 17-76 (mean 47) years diagnosed with major depressive disorder by psychiatric assessment at the Botkin Hospital according to the Diagnostic and Statistical Manual of Mental Disorders, Fourth Edition (DSM-IV). Baseline Clinical Global Impression scores and EIS (electrical conductivity and dispersion $\alpha$ parameter) measurements were done before starting SSRI therapy. Treatment follow-up was undertaken using EIS bioimpedance measurements and by treatment response based on the Hamilton Depression Scale and Clinical Global Impression, every 15 days for 60 days. At day 45, we classified the patients into two groups, ie, Group 1, including treatment responders, and Group 2, including nonresponders. At day 60, patients were classified into two further groups, ie, Group 3, comprising treatment responders, and Group 4, comprising nonresponders.

Results: Comparing Group 1 and Group 2, electrical conductivity measurement of the pathway between the two forehead electrodes had a specificity of $72 \%$ and a sensitivity of $85.3 \%$ $(P<0.0001)$, with a cutoff $>4.32$. Comparing Group 3 and Group 4, electrical conductivity measurements in the same pathway had a specificity of $47.6 \%$ and a sensitivity of $76.3 \%$ $(P<0.16)$, with a cutoff $>5.92$. Comparing Group 1 and Group 2, the electrical dispersion $\alpha$ parameter of the pathway between the two disposable forehead electrodes had a specificity of $80 \%$ and a sensitivity of $85.2 \%(P<0.0001)$ with a cutoff $>0.678$. Comparing Group 3 and Group 4, the electrical dispersion $\alpha$ parameter of the same pathway had a specificity of $100 \%$, a sensitivity of $89.5 \%(P<0.0001)$, and a cutoff $>0.692$.

Conclusion: Electrical conductivity measurement of the forehead pathway using EIS has a high specificity and sensitivity at day 45 when comparing treatment responders and nonresponders, but decreases at day 60 . The EIS electrical dispersion $\alpha$ parameter of the forehead pathway has a high specificity and sensitivity at day 45 when comparing treatment responders and nonresponders, and increases at day 60. The EIS system may be a noninvasive, easily administered, low-cost technique that could be used as an adjunct to DSM-IV and Clinical Global Impression scores for monitoring of efficacy of treatment in patients with major depressive disorder.

Keywords: major depressive disorder, selective serotonin reuptake inhibitors, Electro Interstitial Scan, electrical conductivity, dispersion $\alpha$ parameter

\section{Introduction}

Depression is a common illness with a high degree of morbidity and mortality. ${ }^{1}$ It is a serious disorder that interferes with physical and mental functioning to a greater 
extent than a number of other common chronic conditions, including hypertension, arthritis, and diabetes. ${ }^{1}$ Major depression significantly affects the quality of life and productivity of the patient. Early recognition and treatment are essential to minimize the personal and societal cost associated with depression. ${ }^{2}$ In spite of the serious consequences associated with untreated depression, most studies report that it remains largely underdiagnosed and inadequately treated. ${ }^{2}$ Nevertheless, it is encouraging to note that if treated early and appropriately with an antidepressant, approximately $60 \%-70 \%$ of patients respond to the initial course of therapy. ${ }^{3}$ People respond differently to a variety of treatments, and only Diagnostic and Statistical Manual of Mental Disorders, Fourth Edition (DSM-IV) ${ }^{4}$ and Clinical Global Impression (CGI) scores $^{5}$ are considered to be reliable methods for assessing treatment response. The likelihood of response to a given medication is well known, but psychiatrists are not able to predict if therapy will be effective in an individual patient. In clinical practice, psychiatrists use the method of trial and error. ${ }^{6}$

Bioimpedance has been shown to be a safe technique when used in a number of biomedical applications, including for estimation of body composition, ${ }^{7}$ impedance cardiography, ${ }^{8}$ quantification of brain edema in neurosurgery, ${ }^{9}$ and for detection of differences between normal, abnormal, and malignant prostate tissue. ${ }^{10}$ In this study, we used the Electro Interstitial Scan (EIS) to perform bioimpedance measurements as follow-up of the effect of selective serotonin reuptake inhibitor (SSRI) treatment in patients diagnosed with major depressive disorder. This follow-up was performed from the conductivity and dispersion values of the pathway between two disposable electrodes placed on the forehead and compared with the results of assessment using DSM-IV questionnaire and the CGI scale.

\section{Methods and materials}

This study was approved by the ethics committee at Botkin Hospital, and adhered to the ethical principles of the Declaration of Helsinki. Each patient signed an informed consent form, and confidentiality was maintained for all participants.

\section{Subjects}

Fifty-nine subjects (38 women, 21 men) of mean age 47 (range 17-76) years were recruited from the Psychiatric Department of Botkin Hospital. Patients were excluded if they had a neurological disorder precluding ability to sign a consent form, if in the opinion of the investigator they were clinically unsuitable candidates for the trial, and/or had any contraindications to use of the EIS system. Use of the EIS is contraindicated in the presence of an external defibrillator, skin lesions likely to come into contact with the electrodes, excessive perspiration, a cardiac pacemaker, electronic life support, any implanted electronic device, inability to remain still for three minutes, metallic pins or prostheses in the digits or joints, pregnancy from the third trimester onwards, and absence of a limb.

All 59 patients had a clinical diagnosis of major depressive disorder as scored on the Hamilton Depression ${ }^{11}$ (Ham-D) and CGI scales. Once diagnosed, the patients were sent to the Department of Physiology at Botkin Hospital before starting antidepressant treatment, and an examination was performed using the EIS. The patients were then started on an SSRI (fluoxetine $20 \mathrm{mg} /$ day), and follow-up was undertaken using both EIS bioimpedance measurements and Ham-D and/or CGI scores at 15-day intervals for 60 days. Treatment response was defined as a score of 1 or 2 on the CGI improvement scale and/or a decrease of at least $50 \%$ in Ham-D score. At day 45, patients were classified into two groups, ie, Group 1 (responders) and Group 2 (nonresponders). At day 60, patients were again classified into two groups, ie, Group 3 (responders) and Group 4 (nonresponders).

\section{Materials}

The EIS system is a programmable electromedical system comprising a USB plug and hardware including an interface box, disposable electrodes, reusable plates, and reusable cables, with software installed on a computer. The system uses bioimpedance in bipolar mode with direct current (1.28 V), and measures the electrical conductivity and dispersion $\alpha$ parameter in 11 pathways of the body using six electrodes placed symmetrically on the palms of the hands, soles of the feet, and on the forehead. Each pathway is recorded twice from anode to cathode and then from cathode to anode. Electrode polarization does not affect bioimpedance measurements, ${ }^{12}$ and transmission of the current from the electrode to the hardware is performed by chronoamperometry. ${ }^{13}$

\section{EIS and electrical conductivity}

With direct current, the plasma membrane acts as an insulator and the current is not able to penetrate the cell, so most of the current flows around the cell and therefore in the interstitial fluid. ${ }^{5}$ Analysis of the direct current at the cathode and anode in electrolytic solution is performed at 
Table I Patient demographic data

\begin{tabular}{llllll}
\hline & Group I & Group 2 & Group 3 & Group 4 & P value \\
\hline $\mathrm{n}$ & 34 & 25 & 38 & $2 \mathrm{I}$ & \\
Age & 47.1 & 46.8 & 47.8 & 46.3 & $\mathrm{NS}$ \\
Male/female & 0.54 & 0.48 & 0.55 & 0.46 & $0.0 \mathrm{I}$ \\
ratio & & & & & \\
Conductivity & 4.92 & 3.1 & 6.12 & 3.95 & $0.00 \mathrm{I}$ \\
Dispersion & 0.689 & 0.645 & 0.697 & $0.65 \mathrm{I}$ & $0.00 \mathrm{I}$ \\
Ham-D & $<50 \%$ & $>50 \%$ & $<50 \%$ & $>50 \%$ & \\
CGl average & $\mathrm{I.75}$ & 3 & 1.60 & 3 & \\
\hline
\end{tabular}

Abbreviations: CGI, Clinical Global Impression scale; Ham-D, Hamilton Depression scale; NS, not significant.

both the anode and the cathode. The electrical conductivity is measured in $\mu$ Siemens. In this study, we considered only the pathway between the two disposable frontal electrodes $(\mathrm{Ag} / \mathrm{Ag} / \mathrm{Cl})$. The electrochemical reaction at the cathode ${ }^{12}$ is: $2 \mathrm{H}_{2} \mathrm{O}+2 \mathrm{e}^{-}=\mathrm{H}_{2}$ (gas) $+2 \mathrm{OH}^{-}$(base) and at the anode ${ }^{12}$ is: $2 \mathrm{H}_{2} \mathrm{O}=\mathrm{O}_{2}$ (gas) $+4 \mathrm{H}^{+}+4 \mathrm{e}^{-}($acid $)$.

\section{EIS and electrical dispersion}

The cell membrane has the ability to store capacitive energy via its dielectric or insulator properties. The cell membrane is the cellular structure that makes the major contribution to the dielectric behavior of living tissue. Living tissue is considered as a dispersive medium. ${ }^{13,14}$ Electrical dispersion is expressed by the $\alpha$ parameter value. In 1940, Cole introduced the first mathematical expression to describe the "depressed semicircles" found experimentally. This is known as the Cole equation, as follows:

$$
Z=R_{\infty}+\frac{\Delta R}{1+(j \omega \tau)^{\alpha}}, \quad \Delta R=R_{0}-R_{\infty}
$$

where $Z$ is the impedance value at frequency $\omega, j$ is the complex number $(-1) 1 / 2, R_{\infty}$ is the impedance at infinite frequency, $R_{0}$ is the impedance at zero frequency, $\tau$ is the characteristic time constant, and $\alpha$ is a dimensionless parameter with a value between 0 and $1 .{ }^{13,14}$

\section{Parameters analyzed}

Statistical analysis was conducted to test for concordance between the bioimpedance measurements (electrical conductivity and electrical dispersion $\alpha$ parameter) and treatment responses according to Ham-D and CGI scores. A receiver-operating characteristic curve was constructed for bioimpedance measurements in the pathway of the forehead electrodes for Groups 1 and 2 at day 45, and another receiver-operating characteristic curve was constructed for bioimpedance measurements in the pathway of the forehead electrodes for Groups 3 and 4 at day 60 .

\section{Statistical analysis}

Statistical analysis was performed using MedCalc software. The number of patients needed for the study was calculated to be 50 on the basis of $\alpha=5 \%$, at $80 \%$ power $=\mathrm{F}(\Delta, \mathrm{N}$, variability DS), taking into account the judgment criteria $\Delta$ at approximately $50 \mathrm{DS}$ (5\% error). A $P$ value of $<0.005$ was accepted as being statistically significant.

\section{Results}

Fifty-nine subjects were enrolled in the study and started on SSRI treatment of fluoxetine $20 \mathrm{mg}$ /day.

At day 15, there were no responders to treatment, at day 30 there were six responders, at day 45 there were 34 responders (Group 1) and 25 nonresponders (Group 2), at day 60, there were 38 responders (Group 3) and 21 nonresponders (Group 4).

Electrical conductivity and electrical dispersion increased in the four groups during SSRI treatment. Demographic

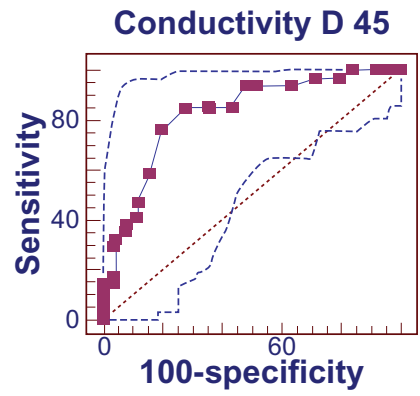

\begin{tabular}{|c|c|c|}
\hline Variable & \multicolumn{2}{|c|}{$\begin{array}{l}\text { Conductivity_D_45 } \\
\text { ConductivityD } 45\end{array}$} \\
\hline Classification variable & \multicolumn{2}{|c|}{ ReponsesD_45 } \\
\hline \multicolumn{2}{|l|}{ Sample size } & 59 \\
\hline \multicolumn{2}{|c|}{ Positive group: ReponsesD_45 $=1$} & 34 \\
\hline \multicolumn{2}{|c|}{ Negative group: ReponsesD_45 $=0$} & 25 \\
\hline \multicolumn{2}{|l|}{ Disease prevalence (\%) } & Unknown \\
\hline \multicolumn{2}{|c|}{ Area under the ROC curve (AUC) } & 0.825 \\
\hline \multicolumn{2}{|l|}{ Standard error ${ }^{15}$} & 0.0568 \\
\hline \multicolumn{2}{|c|}{$95 \%$ confidence interval ${ }^{a}$} & 0.704 to 0.911 \\
\hline \multicolumn{2}{|l|}{ Z statistic } & 5.719 \\
\hline \multicolumn{2}{|c|}{ Significance level $P($ area $=0.5)$} & $<0.0001$ \\
\hline
\end{tabular}

Figure I Comparing Group I (D+45 responders) and Group 2 (D+45 nonresponders), electrical conductivity measurement of the pathway between the two forehead electrodes.

Note: ${ }^{a B i n o m i a l ~ e x a c t . ~}$ 


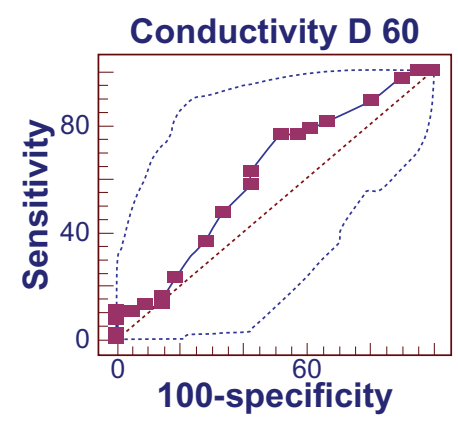

\begin{tabular}{|c|c|c|}
\hline Variable & \multicolumn{2}{|c|}{$\begin{array}{l}\text { Conductivity_D_60 } \\
\text { Conductivity D } 60\end{array}$} \\
\hline Classification variable & \multicolumn{2}{|c|}{$\begin{array}{l}\text { ReponsesD_60 } \\
\text { ReponsesD +60 }\end{array}$} \\
\hline \multicolumn{2}{|l|}{ Sample size } & 59 \\
\hline \multicolumn{2}{|c|}{ Positive group: Reponses $D+60=$} & 38 \\
\hline \multicolumn{2}{|c|}{ Negative group: Reponses $D+60=0$} & 21 \\
\hline \multicolumn{2}{|l|}{ Disease prevalence (\%) } & Unknown \\
\hline \multicolumn{2}{|c|}{ Area under the ROC curve (AUC) } & 0.610 \\
\hline \multicolumn{2}{|c|}{ Standard error ${ }^{15}$} & 0.0798 \\
\hline \multicolumn{2}{|l|}{$95 \%$ confidence interval $^{a}$} & 0.474 to 0.734 \\
\hline \multicolumn{2}{|l|}{ Z statistic } & 1.375 \\
\hline \multicolumn{2}{|c|}{ Significance level $\mathrm{P}($ area $=0.5)$} & 0.1692 \\
\hline
\end{tabular}

Figure 2 Comparing Group 3 ( $D+60$ responders) and Group 4 ( $D+60$ non responders), electrical conductivity measurement of the pathway between the two forehead electrodes.

Note: ${ }^{a}$ Binomial exact.

characteristics are shown in Table 1. Treatment responses were coded as 1 (response) and 0 (no response). Comparing Group 1 and Group 2, electrical conductivity measurement of the pathway between the two forehead electrodes had a specificity of $72 \%$ and sensitivity of $85.3 \%(P<0.0001)$ with a cutoff $>4.32$ (see Figure 1). Comparing Group 3 and Group 4, the electrical conductivity of the same pathway had a specificity of $47.6 \%$ and a sensitivity of $76.3 \%(P<0.16)$ with a cutoff $>5.92$ (see Figure 2). Comparing Group 1 and Group 2, electrical dispersion of the pathway between the two forehead electrodes had a specificity of $80 \%$ and a sensitivity of $85.2 \%(P<0.0001)$ with a cutoff $>0.678$ (Figure 3$)$. Comparing Group 3 and Group 4, electrical dispersion of the same pathway had a specificity of $100 \%$ and a sensitivity of $89.5 \%(P<0.0001)$ with a cutoff $>0.692$ (Figure 4$)$.

\section{Discussion}

People respond differently to antidepressant treatment. With some knowledge of the therapeutic modalities available, both pharmacologic and nonpharmacologic, coupled with a reasonable amount of patience on the part of both the health care provider and the patient, treatment can be highly successful in regaining an appropriate level of well-being. ${ }^{16}$ The antidepressant properties of SSRIs are due to increased concentrations of serotonin at the synaptic cleft, enhancing serotonergic transmission and inducing downregulation of postsynaptic receptors. Although neurotransmitter reuptake inhibition is an important property of SSRIs, the temporal difference between the rapid onset of this pharmacological effect and slower symptomatic relief suggests that secondary adaptive responses may contribute to the effectiveness of SSRIs as antidepressants. ${ }^{17}$

Electroencephalography using disposable frontal electrodes has been investigated in the prediction of response to antidepressant therapy. Various electroencephalographic parameters have been associated with response to treatment. ${ }^{18}$ To our knowledge, this is the first study using the bioimpedance measurement with two components, ie, conductivity

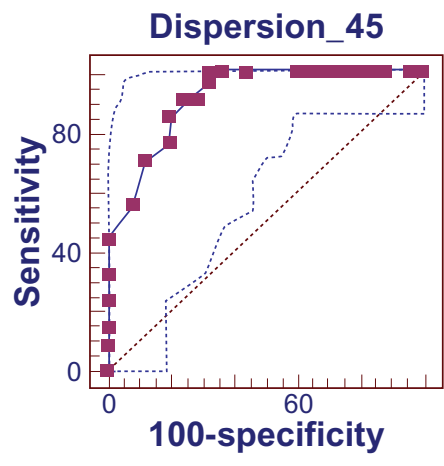

\begin{tabular}{|c|c|c|}
\hline Variable & \multicolumn{2}{|c|}{$\begin{array}{l}\text { Conductivity_45 } \\
\text { ConductivityD_45 }\end{array}$} \\
\hline Classification variable & \multicolumn{2}{|c|}{ ReponsesD_45 } \\
\hline \multicolumn{2}{|l|}{ Sample size } & 59 \\
\hline \multicolumn{2}{|c|}{ Positive group: ReponsesD_45 = 1} & 34 \\
\hline \multicolumn{2}{|c|}{ Negative group: ReponsesD_45 $=0$} & 25 \\
\hline \multicolumn{2}{|l|}{ Disease prevalence (\%) } & Unknown \\
\hline \multicolumn{2}{|c|}{ Area under the ROC curve (AUC) } & 0.914 \\
\hline \multicolumn{2}{|c|}{ Standard error ${ }^{15}$} & 0.0366 \\
\hline \multicolumn{2}{|l|}{$95 \%$ confidence interval $^{a}$} & 0.811 to 0.971 \\
\hline \multicolumn{2}{|l|}{ Z statistic } & 11.311 \\
\hline \multicolumn{2}{|c|}{ Significance level $P($ area $=0.5)$} & $<0.0001$ \\
\hline
\end{tabular}

Figure 3 Comparing Group I (D+45 responders) and Group 2 ( $D+45$ nonresponders), electrical dispersion of the pathway between the two forehead electrodes Note: ${ }^{\text {Binomial exact. }}$ 


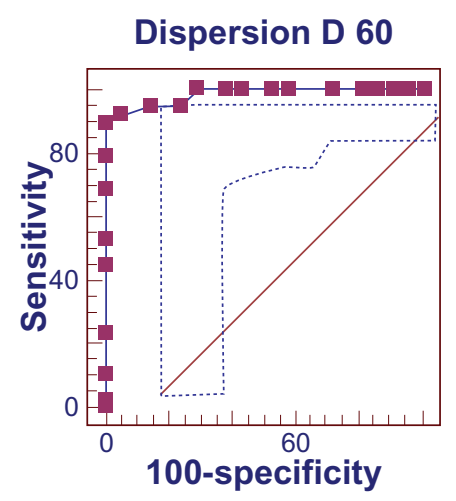

\begin{tabular}{|c|c|c|}
\hline Variable & \multicolumn{2}{|c|}{$\begin{array}{l}\text { Dispersion_D_60 } \\
\text { Dispersion D } 60\end{array}$} \\
\hline Classification variable & \multicolumn{2}{|c|}{$\begin{array}{l}\text { Reponses_60 } \\
\text { Reponses }+60\end{array}$} \\
\hline \multicolumn{2}{|l|}{ Sample size } & 59 \\
\hline \multicolumn{2}{|c|}{ Positive group: Reponses $+60=1$} & 38 \\
\hline \multicolumn{2}{|c|}{ Negative group: Reponses $+60=0$} & 21 \\
\hline \multicolumn{2}{|l|}{ Disease prevalence (\%) } & Unknown \\
\hline \multicolumn{2}{|c|}{ Area under the ROC curve (AUC) } & 0.983 \\
\hline \multicolumn{2}{|c|}{ Standard error ${ }^{15}$} & 0.0117 \\
\hline \multicolumn{2}{|l|}{$95 \%$ confidence interval ${ }^{a}$} & 0.909 to 1.000 \\
\hline \multicolumn{2}{|l|}{ Z statistic } & 41.118 \\
\hline \multicolumn{2}{|c|}{ Significance level P (area = 0.5) } & $<0.0001$ \\
\hline
\end{tabular}

Figure 4 Comparing Group 3 (D+60 responders) and Group 4 ( $D+60$ nonresponders), electrical dispersion of the pathway between the two forehead electrodes.

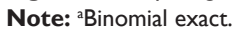

and dispersion, to monitor response to an antidepressant therapy. In our study, the best indicator of treatment response was the dispersion $\alpha$ parameter at day 60 , with a specificity of $100 \%$, a sensitivity of $89.5 \%(P<0.0001)$, and a cutoff $>0.692$.

Ivorra et $\mathrm{al}^{16}$ investigated the significance of the dispersion $\alpha$ parameter by computer simulations and demonstrated its practical importance in a rat kidney model. The simulations indicated that the dispersion width is determined by the morphology of the extracellular space and suggest that this technique could detect structural tissue changes. One explanation as to why electrical conductivity measurements increased in the forehead pathway after 45 days of SSRI treatment could be related to an increased concentration of serotonin at the synaptic cleft and an associated change in cerebral tissue blood flow. A second explanation as why the dispersion $\alpha$ parameter increased in the forehead pathway after 45 and 60 days of SSRI treatment could be related to changes in the morphology of the extracellular space in the cerebral tissue.

\section{Conclusion}

EIS electrical conductivity measurement in the forehead pathway showed high specificity and sensitivity at day 45 for distinguishing between responders and nonresponders to SSRI therapy. The specificity and sensitivity decreased at day 60. The EIS electrical dispersion $\alpha$ parameter in the forehead pathway has a high specificity and sensitivity at day 45 when comparing responders and nonresponders, and the specificity and sensitivity increase at day 60 . EIS could be a low-cost noninvasive system that is easy to use in the office and may become an adjunct to DSM-IV questionnaires and Clinical Global Impression scores for monitoring the efficacy of treatment for major depressive disorder. Longitudinal studies are now under way to confirm our findings.

\section{Disclosure}

The authors report no conflict of interest in this work.

\section{References}

1. Wells KB, Stewart A, Hays RD, et al. The functioning and well-being of depressed patients: results from the Medical Outcomes Study. JAMA. 1989;262:914-919.

2. Montano CB. Recognition and treatment of depression in a primary care setting. J Clin Psychiatry. 1994;55 (Suppl 12):18-34.

3. Wells BG. Issues in the diagnosis of major depression. Formulary. 1995;30 (Suppl 1):S3-S9.

4. American Psychiatric Association. Diagnostic and Statistical Manual of Mental Disorders. Fourth Edition. Washington, DC: American Psychiatry Association; 1994.

5. US Department of Health, Education, and Welfare. Assessment Manual for Psychopharmacology. Rockville, MD: US Department of Health, Education, and Welfare; 1976.

6. Trivedi MH, Kurian BT, Grannemann BD. Clinical predictors in major depressive disorder. Psychiatry Weekly. Available from: http://www. psychiatryweekly.com. Accessed on May 21, 2007.

7. Hamilton M. Rating depressive patients. J Clin Psychiatry. 1980;41:21-24.

8. Rigaud B, Morucci J, Chauveau N. Bioelectrical impedance techniques in medicine. Part I: bioimpedance measurement. Second section: impedance spectrometry. Crit Rev Biomed Eng. 1996;24: 257-351.

9. Woltjer HH, Bogaard HJ, de Vries JM. The technique of impedance cardiography. Eur Heart J. 1997;18;1396-1403.

10. Ko HW, Smith DG, Skura JP. In vitro measurements of brain edema with the magnetic bio-impedance method. Presented at the annual conference of the IEEE Engineering in Medicine and Biology Society, Amsterdam, The Netherlands, October 31-November 3; 1996.

11. De Abreu DS. Bioimpedance and chronoamperometry as an adjunct to prostate-specific antigen screening for prostate cancer. Cancer Manag Res. 2011;3:109-116.

12. Grimmes S, Martinsen ØG. Electrolytics in Bioimpedance and Bioelectricity Basics. San Diego, CA: Academic Press; 2000.

13. Cottrell FG. Application to the Cottrell equation to chronoamperometry. Z Physik Chem. 1902;42:385.

14. Cole KS, Cole RH. Dispersion and absorption in dielectrics. I. Alternatingcurrent characteristics. J Chem Phys. 1941;9:341-351. 
15. DeLong ER, DeLong DM, and Clarke-Pearson DL. Comparing the areas under two or more correlated receiver operating characteristic curves: a nonparametric approach. Biometrics. 1988;44:837-845.

16. Ivorra A, Genescà M, Sola A, et al. Bioimpedance dispersion width as a parameter to monitor living tissues. Physiol Meas. 2005;26:1-9.

17. Song F, Freemantle N, Sheldon TA, et al. Selective serotonin reuptake inhibitors: meta-analysis of efficacy and acceptability. BMJ. 1993;306: 683-687.
18. Fabre LF, Putman HP. A fixed-dose clinical trial of fluoxetine in outpatients with major depression. J Clin Psychiatry. 1987;48:406-408.

19. Bruder GE, Stewart JW, Tenke CE, et al. Electroencephalographic and perceptual asymmetry differences between responders and nonresponders to an SSRI antidepressant. Biol Psychiatry. 2001;49:416-425.

\section{Publish your work in this journal}

Psychology Research and Behavior Management is an international, peerreviewed, open access journal focusing on the science of psychology and its application in behavior management to develop improved outcomes in the clinical, educational, sports and business arenas. Specific topics covered include: Neuroscience, memory \& decision making; Behavior modification \& management; Clinical applications; Business \& sports performance management; Social and developmental studies; Animal studies. The manuscript management system is completely online and includes a quick and fair peer-review system. Visit http://www.dovepress. com/testimonials.php to read real quotes from published authors.

Submit your manuscript here: http://www.dovepress.com/psychology-research-and-behavior-management-journal 